\title{
How long do dental restorations last?
}

Asbjørn Jokstad

Associate Editor, Evidence-based Dentistry

\begin{abstract}
A recent UK National Health Service systematic review has attempted to assess the longevity of dental restorations in general dental practice. The rather disappointing conclusion is that the dental literature does not give any clear indications. Moreover, the research team identified many problems during the preparation of this review that should raise concern both in the dental research community and within research funding agencies. ${ }^{1}$
\end{abstract}

Evidence-Based Dentistry (2002) 3, 89-90. doi:10.1038/sj.ebd.6400138

Why the question cannot be answered warrants some remarks.

- The great majority of studies in restorative dentistry are conducted in vitro.

- The reporting of clinical trials is often poor.

- Trials are mostly done in rigorous clinical settings or using specific outcomes.

- Who wants to know?

The reason for the focus on laboratory studies in preference to clinical trials in restorative dentistry is the supposedly very rapid development of new materials. One may question this argument, however, considering that neither amalgam alloys nor the composite organic parts have undergone any fundamental modifications during the last 30 years. A more plausible explanation is that laboratory data can be amassed quickly and cheaply for publication. This may or may not be used by a manufacturer for promoting its product, whereas a clinical trial involving patients can present ethical, logistic and financial challenges and involve problems with infrastructure.

It should be borne in mind that repeated assessments have demonstrated very poor correlations between in-vivo and in-vitro results. ${ }^{2}$ Dental professionals should therefore continuously demand relevant clinical data from manufacturers when new products are being promoted, rather than assuming that "somebody out there" checks that promotional material and that assertions of clinical excellence are correct. The sad fact is that there is not somebody out there who does this assessment.

There is always an element of judgement involved when the substance of a paper is evaluated, but the critical reader and study methodologists are stuck with pure guesswork when reading many of the papers in the scientific dental literature. This situation is not limited to restorative dentistry. There is a real need for this issue to be addressed by all stakeholders, among members of academia, dental journal editors and content experts who are editorial board members and referees. If an excellent clinical trial has been conducted according to the highest standards, inadequate reporting will inevitably erode the scientific impact whether justified or not. It is astounding therefore that guidelines for adequate conduct and reporting of clinical trials - as suggested for example by CONSORT ${ }^{3}$ and others ${ }^{1}$ - are so seldom followed in dental journals.
Numerous clinical studies were excluded in the NHS review. These exclusions do not mean that they are all flawed, but rather that they do not provide answer to the specified question about longevity. It is a common mistake to interpret excluded studies in systematic reviews as inferior, which is not always correct. There are many first-rate trials that have used a splitmouth randomised design that provides answers to multiple questions, but not specifically on longevity. Other excellent clinical studies were not included in the present review because they only focused on, for example, marginal degradation, surface wear, luting cement dissolution, bulk or marginal discoloration, post-operative sensitivity, adaptation or other clinical outcomes that did not require replacements. In summary, the research in restorative dentistry seems to be primarily aimed to improve materials rather than to document performance in the hands of the general practitioners.

As the question of dental restoration longevity is highly relevant for society, for dental health-care providers and for third-party payers, as well as for many individuals, how is it possible that we cannot provide a precise answer to the question? The answer can be deduced by asking who has provided funding to enable dental researchers to carry out such studies?

To my knowledge, there are no publications of large longitudinal studies sponsored by any insurance or equivalent companies or third-party enterprises. This does not automatically mean that such studies never have been undertaken. It seems reasonable to 
assume that these companies carry out longitudinal studies to generate data as a basis for estimates of their premiums and returns. As such studies involve substantial costs and generate important business-related data they will not be disclosed to the public. The bottom line is that business life is not about improving oral health but to generate profits for shareholders.

The manufacturers sponsor trials that primarily demonstrate the clinical potential of new materials and products. It is not in their interest to support studies that show how their products function in the hands of a spectrum of general practitioners, which seem almost certain to produce worse results than a well-controlled clinical trial. The bottom line here is again just as stated above, although companies' short-term and long-term goals make the issue of relationship between dental manufacturers and researchers slightly more subtle.

Society as a whole is left as the sole realistic source for potential future studies that will generate data describing the long-term clinical performance of dental restorations. The financial costs and logistics associated with a prospective controlled longitudinal study over 10 years, randomised or not, are probably beyond the reach of any dental teaching institution. This situation will require dedicated financial support to prioritise the issue because restorative dentistry is not ranked high-priority in the competitive market for medical research funding.
Without the political will to devote funding for this purpose, we will have to wait for a long time for the studies we need to evaluate the longevity of dental restorations.

1. Chadwick B, Treasure E, Dummer P, et al. Challenges with studies investigating longevity of dental restorations a critique of a systematic review. J Dentistry 2001; 29:155-161.

2. Wilson NHF. The evaluation of materials: relationship between laboratory investigations and clinical studies. Operative Dentistry 1990; 15:149-155.

3. Moher D, Schulz KF, Altman DG, CONSORT Group. The CONSORT statement: revised recommendations for improving the quality of reports of parallelgroup randomised trials. J Am Med Assoc 2001; 285:1987-1991. 